\title{
Decreased plasmatic spermidine and increased spermine in mild cognitive impairment and Alzheimer's disease patients
}

\author{
Helena P. G. Joaquim* ${ }_{1,2}$ \\ https://orcid.org/0000-0003-3966-6072 \\ Alana C. Costa*1,2 \\ Orestes V. Forlenza ${ }^{1,2}$ \\ https://orcid.org/0000-0002-6962-5899 \\ Wagner F. Gattaz ${ }^{1,2}$ \\ https://orcid.org/0000-0001-5215-4077 \\ Leda L. Talib ${ }^{1,2}$ \\ https://orcid.org/0000-0001-5866-1993 \\ 1 Laboratory of Neuroscience (LIM-27), Department and Institute of Psychiatry, University of Sao Paulo, SP, Brazil. \\ ${ }^{2}$ National Institute of Biomarkers in Neuropsychiatry (INBioN), National Council for Scientific and Technological Development (CNPq), São Paulo, SP, Brazil. \\ * Helena P. G. Joaquim and Alana C. Costa contributed equally to this work.
}

Received: 1/24/2019 - Accepted: 4/30/2019

DOl: 10.1590/0101-60830000000209

\begin{abstract}
Background: Current evidence suggests that upregulation of polyamines system plays a role both in cognitive deficit and synaptic loss observed in Alzheimer's disease $(\mathrm{AD})$. Objective: The aim of this study was to determine the plasmatic concentration of polyamines in mild cognitive impairment (MCI) and $\mathrm{AD}$ patients in comparison with healthy controls (HC). Methods: Plasmatic polyamines were quantified using the AbsoluteIDQ ${ }^{\circ}$ p 180 and liquid chromatography coupled to tandem mass spectrometry (LC/MS-MS). Results: The study group comprised $34 \mathrm{AD}$ patients, $20 \mathrm{MCI}$ and $25 \mathrm{HC}$. All individuals were followed for 4 years. During this period 8 amnestic MCI patients (40\% of the MCI sample at baseline) converted to AD. Spermidine level was lower in both patient groups (AD; MCI) compared to HC ( $\mathrm{p}=0.007)$. Plasma levels of spermine were higher in the MCI group $(\mathrm{p}<0.001)$, but decreased in the sub-sample of MCI patients who converted to $\mathrm{AD}(\mathrm{p}=0.043)$. No statistically significant differences were found in ornithine and putrescine levels ( $\mathrm{p}=0.056$ and $\mathrm{p}=0.126$, respectively). Discussion: Our results suggest dynamic changes in the expression of polyamines in the MCI-AD continuum.
\end{abstract}

Joaquim HPG et al. / Arch Clin Psychiatry. 2019;46(5):120-4

Keywords: Biomarkers, Alzheimer's disease, mild cognitive impairment, polyamines, plasma.

\section{Introduction}

The clinical diagnosis of Alzheimer's disease (AD) is a probabilistic definition that takes into account the patient's complaints, the objective characterization of cognitive impairment, family history of dementia, neuropsychiatric examination, laboratory tests and brain imaging ${ }^{1,2}$. Brain imaging and cerebrospinal fluid (CSF) levels of betaamyloid peptide 42 (A $\beta 42)$, Tau and phosphorylated Tau can help in differential diagnosis of $\mathrm{AD}$ and can be useful for predicting $\mathrm{AD}$ in individuals with mild cognitive impairment (MCI) $)^{3,4}$. The search for biological markers in $\mathrm{AD}$ and related disorders is important for a better understanding of the pathophysiological process, and the clinical translation of this knowledge can provide support to the diagnostic workup and the prediction of dementia in patients with mild cognitive symptoms. New technologies have raised the possibility of using biological markers to reinforce the diagnosis of probable $\mathrm{AD}$ at early stages of the disease process ${ }^{5,6}$, addressing in vivo molecules related to core pathogenic mechanisms of $\mathrm{AD}$ and to surrogate markers of the neurodegenerative process, i.e., related to the formation of neuritic plaques and neurofibrillary tangles. Neuritic plaques are extracellular lesions and their main constituent is the amyloid- $\beta 42$ peptide (A $\beta 42)$. Neurofibrillary tangles are intracellular lesions and are mostly composed of hyperphosphorylated TAU protein ${ }^{7-9}$. Both are commonly referred to as 'molecular' markers of the disease process ${ }^{10,11}$.

Polyamines (PAs) are low-molecular-weight organic compounds that perform important functions in cell growth and proliferation, gene transcription, protein synthesis, conformation of nucleic acid and apoptosis ${ }^{12,13}$. The biosynthesis of natural PA initiates from two amino acids, arginine (Arg) and ornithine (Orn). Orn is decarboxylated by ornithine decarboxylase (ODC), the limiting enzyme in the PA synthesis, first to putrescine, which is the immediate precursor for the synthesis of the spermidine and spermine. In mammalian cells, the main natural PAs are putrescine, spermidine and spermine ${ }^{14}$ that are localized inside and outside of cells, as well as in the plasma membrane (Figure 1)15,16.

The effects of abnormal increases in the concentration of neural PAs are not clear. Nevertheless, current evidence suggests that upregulation of PA system plays a role in both cognitive deficit and synaptic loss observed in $\mathrm{AD}^{17}$. It appears that PAs and $\mathrm{A} \beta$ have several potential interaction sites in vivo, particularly in cellular organelles, in the cytoplasm and inside neuronal cells ${ }^{18}$. A $\beta$ peptides have been shown to be responsible for upregulated PA metabolism, specifically, increased PA uptake and high ODC activity ${ }^{19,20}$. Also, PAs are critically involved in microtubule assembly and stabilization ${ }^{21-23}$. Recently, Vemula et al. ${ }^{24}$, determined the time course of the arginine metabolic profile changes in the frontal cortex and hippocampus (among others) when TAU synthesis is increased. They speculate a shift of L-arginine metabolism to favor the polyamine as a protective mechanism. In addition, spermidine and spermine have the ability to modulate several ion channels in the brain specifically related to the N-methyl-D-aspartate (NMDA) receptor ${ }^{25,26}$. Dysfunction of the NMDA receptor, established as neuronal excitotoxicity is hypothesized to be involved in the etiology of $\mathrm{AD}^{27,28}$. 


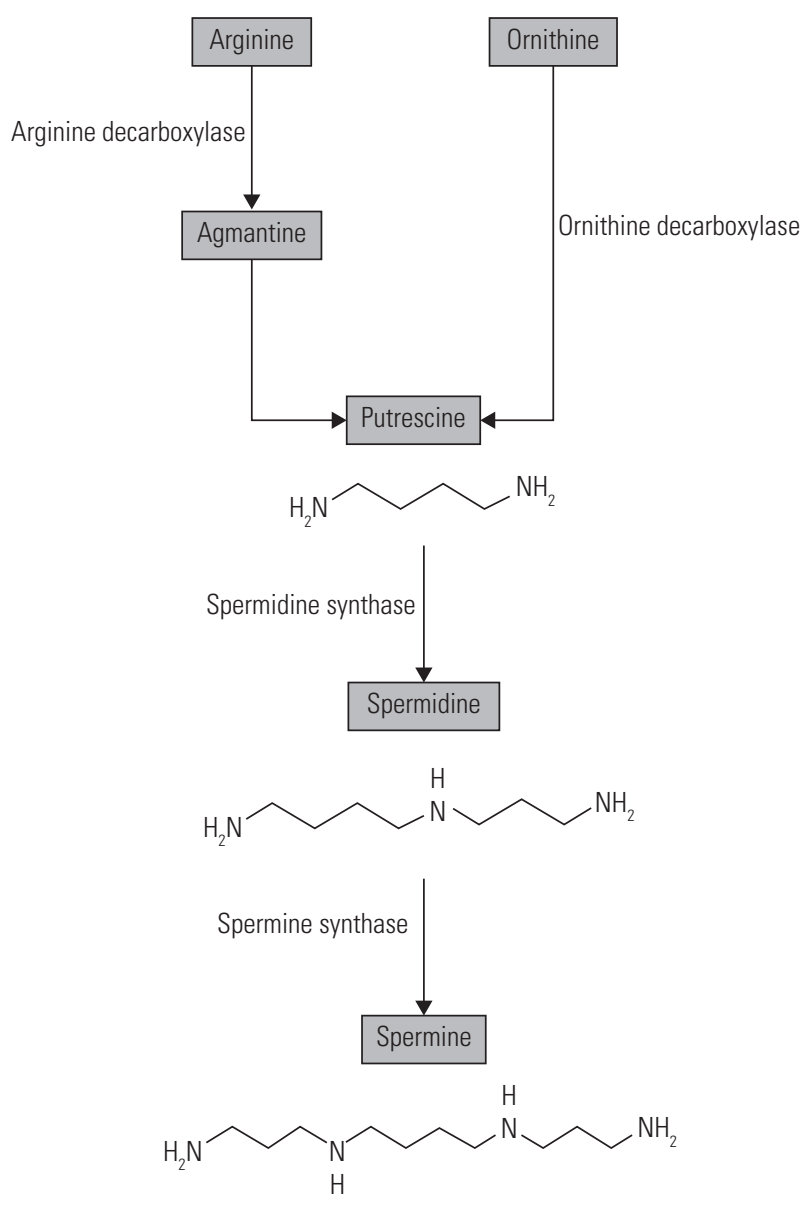

Figure 1. Polyamines structures and biosynthesis pathway.

Exploring the role of polyamines in the pathophysiology of $\mathrm{AD}$ and its prodromal phase MCI represents an alternative to developing biomarkers. Thus, the aim of this study was to determine the plasmatic concentration of PAs in MCI and AD patients compared to healthy controls.

\section{Material and methods}

\section{Subjects}

Eligible subjects were recruited from a cohort of community-dwelling older adults attending a psychogeriatric outpatient facility at the Institute of Psychiatry, Faculty of Medicine, University of São Paulo, Brazil. Participants, mostly from the hospital catchment area, were either outpatient undergoing treatment for cognitive disorders or healthy volunteers in a longitudinal follow-up study conducted at the memory clinic. Seventy nine subjects were enrolled in this study: $34 \mathrm{AD}, 20$ amnestic MCI and 25 physically and cognitively healthy controls (Table 1). In the last 6 months prior to enrolment in the study, patients and the control group did not use AChEi or other drugs (e.g. neuroleptics, antidiabetic, lithium, antidepressants and anticonvulsants). Subjects with other psychiatric or neurologic diseases and others dementias were excluded. The diagnosis of mild to moderate $\mathrm{AD}$ was established according to the NINCDS-ADRDA diagnostic criteria ${ }^{29}$. The clinical condition of MCI was determined according to the Petersen criteria ${ }^{30}$. Blood samples were collected at baseline. The Cambridge Cognitive Test (CAMCOG) ${ }^{31}$ and MiniMental State Examination (MMSE) ${ }^{32}$ assessed at baseline and at oneyear intervals cognitive impairment. Obviously, the cognitive scores were different between the groups, with decreased performance in $\mathrm{MCI}$ and in $\mathrm{AD}$ patients (Table 1).
Table 1. Socio-demographic characteristics of patients and controls

\begin{tabular}{|l|c|c|c|c|}
\hline Diagnosis & $\begin{array}{c}\mathrm{AD} \\
(\mathrm{n}=34)\end{array}$ & $\begin{array}{c}\mathrm{MCl} \\
(\mathrm{n}=20)\end{array}$ & $\begin{array}{c}\mathrm{HC} \\
(\mathrm{n}=25)\end{array}$ & $p$ \\
\hline Gender (M/F) & $10 / 24$ & $3 / 17$ & $7 / 18$ & 0.47 \\
\hline Age (mean $\pm \mathrm{sd})$ & $75.0 \pm 6.7$ & $73.9 \pm 6.2$ & $74.4 \pm 5.8$ & 0.81 \\
\hline Years of education (mean $\pm \mathrm{sd})$ & $6.2 \pm 3.8$ & $7.8 \pm 4.4$ & $13.2 \pm 5.7$ & 0.01 \\
\hline MMSE (mean $\pm \mathrm{sd})$ & $19.0 \pm 5.0$ & $27.0 \pm 2.0$ & $29.0 \pm 1.0$ & $<0.01$ \\
\hline CAMCOG (mean $\pm \mathrm{sd})$ & $57.0 \pm 18.0$ & $86.0 \pm 9.0$ & $95.0 \pm 6.0$ & $<0.01$ \\
\hline
\end{tabular}

M: male; F: female; sd: standard deviation; AD: Alzheimer's disease; MCl: mild cognitive impairment; HC: healthy control; MMSE: Mini-Mental State Examination; CAMCOG: Cambridge Cognitive Test. Bold values indicate significant p-values.

All individuals were followed during 4 years. During this period, 8 amnestic MCI patients converted to AD (converters MCI-AD) and 12 did not (MCI-NC). These eight MCI patients performed new blood collection after conversion.

The study was approved by the Local Ethics Committee of University of Sao Paulo (CAPPesq $\mathrm{n}^{\circ}$ 943.883) and performed in accordance with the Helsinki declaration. All subjects provided written informed consent prior to inclusion in the study. The sociodemographic characteristics of the patients and controls are summarized in Table 1. All results were controlled for years of education and gender because of statistical differences.

\section{Sample preparation}

Blood samples of all subjects were collected in EDTA-coated tubes (Vacuntainer, Bencton Dickison) for plasma metabolite determination after 8 hours of fasting. Samples were centrifuged at $20^{\circ} \mathrm{C}$ and $1,800 \mathrm{~g}$ for $15 \mathrm{~min}$ and stored at $-80^{\circ} \mathrm{C}$ until analysis.

\section{Polyamines quantification}

We analyzed endogenous metabolites with an AbsoluteIDQ p180 kit (Biocrates Life Science AG, Innsbruck, Austria), and performed a targeted quantitative and quality controlled assay using liquid chromatography coupled to tandem mass spectrometry (LC-MS/ MS). In brief, after the addition of $10 \mathrm{uL}$ if the supplied internal standard solution to each well on a filterspot of the 96-well extraction plate, $10 \mathrm{uL}$ of each plasma sample, quality control samples, blank, zero sample, or calibration standard were added to the appropriate wells. The plate was then dried under a gentle stream of nitrogen. The samples were derivatized with phenyl isothiocyanate (PITC) for the biogenic amines, and dried again. Sample extracts were diluted with either $40 \%$ methanol in water for the LC-MS/MS analysis. This technique uses isotope-labelled internal standards and provides quantitative results based on calibration curves and quality control analyses. Low/mid/high level QC samples provided by Biocrates Life Science AG were prepared and analyzed on each plate as recommended by the manufacturer. These QC samples were used for a technical validation of each kit plate. To allow appropriate inter-plate abundance scaling based specifically on this cohort of samples, we generated a Study Pool QC by combining approximately $10 \mu \mathrm{l}$ from the first 20 samples for analysis. This sample was frozen and analyzed on each plate. This analysis was performed on a triplequadrupole mass spectrometer (Xevo TQ-S, Waters Corporation, USA), using positive electrospray ionization operating in the Multiple Reaction Monitoring (MRM) mode. MRM transitions (compoundspecific precursor to product ion transitions) for each analyte and internal standard were collected over a scheduled retention time window using tune files and acquisition methods provided in the AbsoluteIDQ p180 kit. The plasma samples were processed according manufacturer instructions. Chromatographic separation of biogenic amines was performed using an ACQUITY UPLC System (Waters Corporation) using an ACQUITY $2.1 \mathrm{~mm} \times 50 \mathrm{~mm} 1.7 \mu \mathrm{m}$ BEH C18 column fitted with an ACQUITY BEH C18 1.7 $\mu \mathrm{m}$ VanGuard guard 
column, and quantified by calibration curve plotting ratio of analyte to internal standard versus standard concentration, fitted using a linear regression with $1 / x$ weighting. All biogenic amines utilize either deuterated or $13 \mathrm{C}$ stable-isotope labeled internal standard of the exact analyte or closely-eluting compound of similar class. MassLynx v4.1 software (Waters Technologies) was used to calculate the concentrations of metabolites, peak integration and calibration. The data from MassLynx were analyzed using Biocrates' MetIDQ v5.4.8 software.

\section{Statistical analysis}

We performed the statistical analysis using the software SPSS v.22 (Statistical Package for Social Science, Chicago, IL). For gender distribution analysis, we used Chi-square test. We checked normality assumptions with QQ plots. For variables with normal distribution we analyzed by ANOVA test; Kruskal-Wallis test for variables with non-normal distribution; Dunn-Bonferroni for pairwise comparison. We used Wilcoxon test to compare longitudinally MCI patients that have converted to AD. The statistical significance adopted for all analyses was $p \leq 0.05(\alpha=95 \%)$.

\section{Results}

Plasmatic concentrations of spermidine were lower in patients with any degree of cognitive impairment ( $\mathrm{AD}$ and $\mathrm{MCI}$ ) as compared to healthy controls $(\mathrm{p}=0.007)$ (Figure 2$)$.

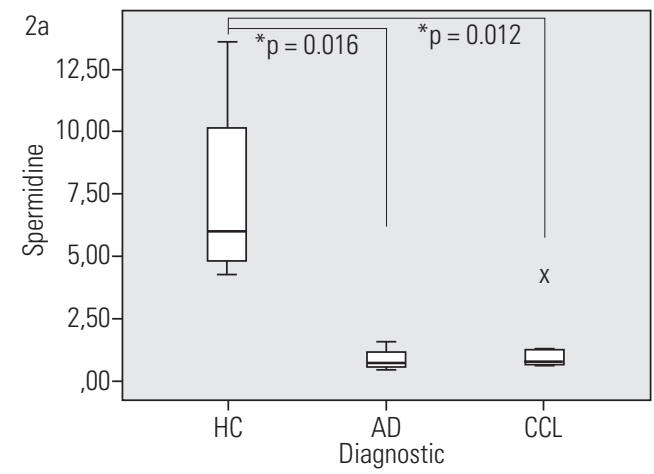

Figure 2a. Concentration of PAs metabolites in $A D$ and $\mathrm{MCl}$ patients - Levels of spermidine in $A D$ and $\mathrm{MCl}$ patients are lower than in $\mathrm{HC}$. $\mathrm{p}$ : significance of post-hoc tests; $\mathrm{AD}$ : Alzheimer's disease; $\mathrm{MCl}$ : mild cognitive impairment; $\mathrm{HC}$ : healthy controls.

Spermine levels were higher in the MCI group compared to the other two groups $(\mathrm{p}=<0.001)$.

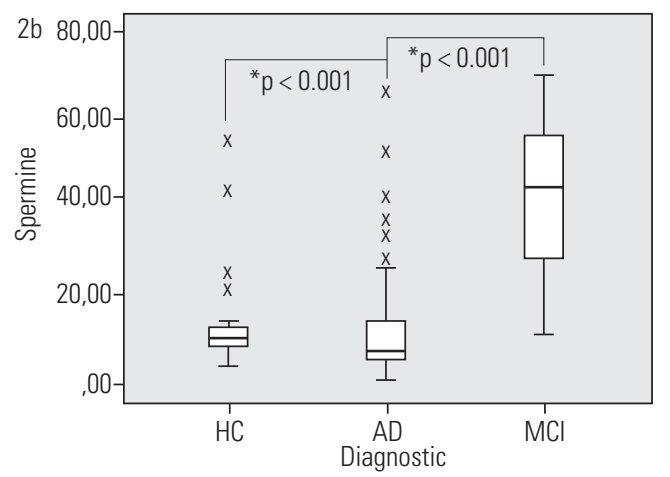

Figure $\mathbf{2 b}$. Concentration of PAs metabolites in $\mathrm{AD}$ and $\mathrm{MCl}$ patients Spermine levels in $\mathrm{MCl}$ patients are 3 times higher than in $\mathrm{AD}$ patients and HC. p: significance of post-hoc tests; AD: Alzheimer's disease; MCl: mild cognitive impairment; $\mathrm{HC}$ : healthy controls.
The MCI patients grouped in MCI who subsequently converted in AD (MCI_AD) and MCI no converters presented no statistical differences in polyamines levels, however, the sub-sample of MCI patients who progressed to dementia upon follow-up also had lower plasma concentrations of spermine at baseline (Figure 3).

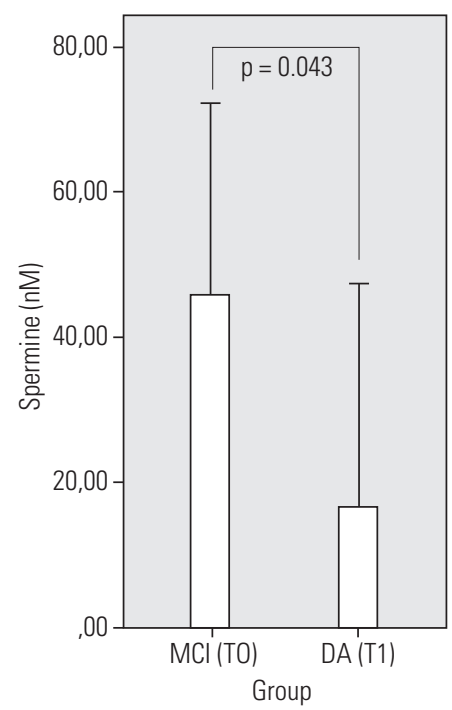

Figure 3. Concentration of plasmatic spermine in 8 patients whom converted from $\mathrm{MCl}$ (TO) to $\mathrm{AD}$ (T1) after 4 years' clinical follow-up).

No statistically significant differences were found in plasmatic levels of arginine, ornithine and putrescine between the three groups (Table 2). The difference in frequency of participant's gender was the same in all groups $(p=0.47$; Table 1$)$. There was also no association with any polyamines' levels and gender. Either way, the results were controlled by gender and years of education.

\section{Discussion}

In this study, we determined plasmatic levels of the amino acids arginine and ornithine, and the polyamines putrescine, spermidine and spermine, searching for differences in the concentrations of these amine-containing molecules in patients with mild and severe cognitive impairment (i.e., MCI and AD) compared to healthy, age-matched controls. We found no alteration either on arginine, ornithine or putrescine. Mean spermine concentrations were three times higher in the MCI group as compared to controls and AD but, interestingly, these levels were actually decreased in the sub-sample of MCI patients who converted to AD. Spermidine levels were also lower in $\mathrm{AD}$ and $\mathrm{MCI}$ groups when compared to healthy controls.

The above findings are in agreement with the emerging evidence from studies supporting changes in PAs in cognitive/ neurodegenerative disorders such as $\mathrm{AD}$ and its prodromal phase MCI. Brain metabolic profiling studies also indicate that changes in $\mathrm{PAs}$ pathway do occur in $\mathrm{AD}$, along with an increment of spermidine and spermine in localized brain regions as frontal lobe and parietal

Table 2. Concentration (nmol/L) of polyamine pathway metabolites in $A D$ and $\mathrm{MCl}$ patients

\begin{tabular}{|l|c|c|c|c|}
\hline Metabolite & $\mathrm{AD}$ & $\mathrm{MCl}$ & $\mathrm{HC}$ & $p$ \\
\hline Arginine & $69.62 \pm 18.04$ & $63.02 \pm 1871$ & $80.52 \pm 44,64$ & 0.111 \\
Ornithine & $64.07 \pm 19.42$ & $59.34 \pm 18.47$ & $79.84 \pm 49.15$ & 0.056 \\
\hline Putrescine & $0.08 \pm 0.07$ & $0.14 \pm 0.05$ & $0.43 \pm 1.07$ & 0.126 \\
\hline Spermidine & $0.90 \pm 0.48$ & $1.54 \pm 1.51$ & $7.47 \pm 4.18$ & 0.007 \\
\hline Spermine & $14.34 \pm 15.73$ & $41.71 \pm 18.33$ & $13.13 \pm 11.28$ & $<0.001$ \\
\hline
\end{tabular}

AD: Alzheimer's disease; $\mathrm{MCl}$ : mild cognitive impairment; $\mathrm{HC}$ : healthy controls. Data are presented as mean \pm standard deviation. Bold values indicate significant $p$-values. 
lobe; putrescine levels were increased in frontal lobe ${ }^{31}$. Further, changes in concentrations of plasmatic PAs have also been described. Graham et al. proposed that these changes might help predict the conversion to dementia in MCI patients up to 2 years before the onset of functional deficits ${ }^{33}$. Their study showed that MCI subjects who subsequently developed dementia (AD) present higher plasmatic PAs levels as compared to stable cases of $\mathrm{MCI}^{33}$. Our study corroborates Graham et al., since we found an increased PAs level 4 years before the onset in a small subset of MCI_AD patients.

Luo et al. showed that PAs might interact with amyloid-beta $(A \beta)$ promoting its fibrillation and reducing the neurotoxicity of $\mathrm{A} \beta$ peptides ${ }^{34}$. Hence, PAs, especially spermine, may interact with toxic $A \beta$ forms promoting the structural transition of $A \beta$ toward less toxic fibrillary conformations. This increase of spermine level might be a physiological attempt to decrease neurotoxicity of $A \beta 18,34$. In accordance with these findings, our results suggest that pathological process in $\mathrm{AD}$ is associated with depletion of protective PAs spermine and spermidine, given that the plasmatic concentrations of these two molecules are reduced both at pre-dementic and dementic stages of the MCI-AD continuum. Notwithstanding, it is possible that adaptative responses may upregulate the expression of the former, leading to a temporary, but significant three-fold increase in the expression of spermine in MCI. We hypothesize that, in the presence of continuous/progressive $A \beta$ toxicity, the exhaustion of this adaptative response may ultimately lead decrease in the availability of this $\mathrm{PA}^{35}$.

There are few metabolomics studies with plasma reporting polyamine changes in $\mathrm{AD}$ patients. The findings regarding putrescine and spermidine plasmatic levels are still inconclusive in AD and $\mathrm{MCI}^{33}$. Our results do not show any statistically significant differences in putrescine levels between the three groups. However, it has been reported that stable MCI subjects present higher plasmatic putrescine levels ${ }^{33}$. Although putrescine is increased in certain brain regions of $\mathrm{AD}$ patients, in plasma it seems not to accumulate because it is preferentially uptaken as a precursor for the synthesis of the spermidine and, subsequentially, the spermine ${ }^{33,27}$. Spermidine in plasma has been reported to be decreased in stable MCI patients, but not in MCI who afterwards developed to $\mathrm{AD}$, which was interpreted as possible predictor of conversion to $\mathrm{AD}^{33}$.

The small sample size from each diagnostic group is an important limitation of the study, therefore the results should be replicated in an independent and larger sample to reinforce these findings. In addition, it is necessary to validate these findings in other sample cohorts.

\section{Conclusions}

Interestingly, our results suggest dynamic changes in the expression of spermine in the MCI-AD continuum, which reinforce the need for additional studies in this field aiming at a better understanding of the role of these important mediators in the pathophysiology of AD.

\section{Author contributions}

Authors ACC, HPGJ and LLT managed the literature searches. ACC and HPGJ undertook the statistical analysis. ACC wrote the first draft of the manuscript. Authors ACC and HPGJ wrote the protocol and performed the laboratory analyses. Authors OVF was responsible for clinical assessment. Authors LLT and WFG designed the study. All authors contributed and approved the final manuscript.

\section{Conflict of interest}

The authors declare no conflict of interest.

\section{Acknowledgements}

We are thankful to Danilo Pereira of the Waters Corporation, who performed the assay analyses.

\section{Funding}

This study was supported by the Fundação de Amparo à Pesquisa do Estado de São Paulo - Fapesp (Grants no 2014/20913-3 and 2013/103509), Instituto Nacional de Biomarcadores em Neuropsiquiatria (INBioN - Grant No. 2014/50873-3). The Laboratory of Neuroscience receives financial support from Associação Beneficente Alzira Denise Hertzog da Silva (ABADHS).

\section{References}

1. Barthel H, Gertz HJ, Dresel S, Peters O, Bartenstein P, Buerger K, et al.; Florbetaben Study Group. Cerebral amyloid- $\beta$ PET with florbetaben $(18 \mathrm{~F})$ in patients with Alzheimer's disease and healthy controls: a multicentre phase 2 diagnostic study. Lancet Neurol. 2011;10(5):424-35.

2. McKhann GM, Knopman DS, Chertkow H, Hyman BT, Jack CR Jr, Kawas $\mathrm{CH}$, et al. The diagnosis of dementia due to Alzheimer's disease: Recommendations from the National Institute on Aging-Alzheimer's Association workgroups on diagnostic guidelines for Alzheimer's disease. Alzheimers Dement. 2011;7(3):263-9.

3. Forlenza OV, Diniz BS, Talib LL, Radanovic M, Yassuda MS, Ojopi $\mathrm{EB}$, et al. Clinical and biological predictors of Alzheimer's disease in patients with amnestic mild cognitive impairment. Rev Bras Psiquiatr. 2010;32(3):216-22.

4. Forlenza OV, Radanovic M, Talib LL, Aprahamian I, Diniz BS, Zetterberg $\mathrm{H}$, et al. Cerebrospinal fluid biomarkers in Alzheimer's disease: Diagnostic accuracy and prediction of dementia. Alzheimers Dement (Amst). 2015;1(4):455-63.

5. Henriksen K, O’Bryant SE, Hampel H, Trojanowski JQ, Montine TJ, Jeromin A, et al. The future of blood-based biomarkers for Alzheimer's disease Alzheimers Dement. 2014;10(1):115-31.

6. O'Bryant SE, Mielke MM, Rissman RA, Lista S, Vanderstichele H, Zetterberg H, et al. Blood-based biomarkers in Alzheimer disease: Current state of the science and a novel collaborative paradigm for advancing from discovery to clinic. Alzheimers Dement. 2017;131():45-58.

7. Bussière T, Giannakopoulos P, Bouras C, Perl DP, Morrison JH, Hof PR. Progressive degeneration of nonphosphorylated neurofilament protein-enriched pyramidal neurons predicts cognitive impairment in Alzheimer's disease: Stereologic analysis of prefrontal cortex area 9. J Comp Neurol. 2003;463(3):281-302

8. Qin W, Ho L, Wang J, Peskind E, Pasinetti GM. S100A7, a novel Alzheimer's disease biomarker with non-amyloidogenic alpha-secretase activity acts via selective promotion of ADAM-10. PLoS One. 2009;4(1):e4183.

9. Yoon SS, Jo SA. Mechanisms of Amyloid- $\beta$ Peptide Clearance: Potential Therapeutic Targets for Alzheimer's Disease. Biomol Ther (Seoul). 2012;20(3):245-55.

10. Blennow K, Dubois B, Fagan AM, Lewczuk P, de Leon MJ, Hampel H. Clinical utility of cerebrospinal fluid biomarkers in the diagnosis of early Alzheimer's disease. Alzheimers Dement. 2015;11(1):58-69.

11. Jack CR Jr, Knopman DS, Jagust WJ, Shaw LM, Aisen PS, Weiner MW, et al. Hypothetical model of dynamic biomarkers of the Alzheimer's pathological cascade. Lancet Neurol. 2010;9(1):119-28.

12. Pegg AE. Functions of Polyamines in Mammals. J Biol Chem. 2016;291(29):14904-12.

13. Marton LJ, Pegg AE. Polyamines as targets for therapeutic intervention. Annu Rev Pharmacol Toxicol. 1995;35:55-91.

14. Mandal S, Mandal A, Johansson HE, Orjalo AV, Park MH. Depletion of cellular polyamines, spermidine and spermine, causes a total arrest in translation and growth in mammalian cells. Proc Natl Acad Sci U S A. 2013;110(6):2169-74

15. Rubinstein S, Breitbart H. Cellular localization of polyamines: Cytochemical and ultrastructural methods providing new clues to polyamine function in ram spermatozoa. Biol Cell. 1994;81(2):177-83.

16. Yatin M. Polyamines in living organisms. J Cell Mol Biol. 2002;1:57- 67.

17. Guerra GP, Rubin MA, Mello CF. Modulation of learning and memory by natural polyamines. Pharmacol Res. 2016;112:99-118.

18. Luo J, Yu CH, Yu H, Borstnar R, Kamerlin SC, Gräslund A, et al. Cellular polyamines promote amyloid- $\beta$ peptide fibrillation and modulate the aggregation pathways. ACS Chem Neurosci. 2013;4:454-62.

19. Bernstein HG, Müller M. Increased immunostaining for L-ornithine decarboxylase occurs in neocortical neurons of Alzheimer's disease patients. Neurosci Lett. 1995;186(2-3):123-6. 
20. Nankai M, Klarica M, Fage D, Carter C. The pharmacology of native $\mathrm{N}$-methyl-D-aspartate receptor subtypes: different receptors control the release of different striatal and spinal transmitters. Prog Neuropsychopharmacol Biol Psychiatry. 1998;22(1):35-64.

21. Savarin P, Barbet A, Delga S, Joshi V, Hamon L, Lefevre J, et al. A central role for polyamines in microtubule assembly in cells. Biochem J. 2010;430(1):151-9.

22. Hamon L, Savarin P, Curmi PA, Pastré D. Rapid assembly and collective behavior of microtubule bundles in the presence of polyamines. Biophys J. 2011;101(1):205-16.

23. Song Y, Kirkpatrick LL, Schilling AB, Helseth DL, Chabot N, Keillor JW, et al. Transglutaminase and polyamination of tubulin: posttranslational modification for stabilizing axonal microtubules. Neuron. 2013;78(1):109-23.

24. Vemula P, Jing Y, Zhang H, Hunt JB Jr, Sandusky-Beltran LA, Lee DC, et al. Altered brain arginine metabolism in a mouse model of tauopathy. Amino Acids. 2019;51(3):513-28.

25. Cacabelos R, Takeda M, Winblad B. The glutamatergic system and neurodegeneration in dementia: preventive strategies in Alzheimer's disease. Int J Geriatr Psychiatry. 1999;14(1):3-47.

26. Takano K, Ogura M, Nakamura, Y, Yoneda Y. Neuronal and glial responses to polyamines in the ischemic brain. 2005;2(3):213-23.

27. Inoue K, Tsutsui H, Akatsu H, Hashizume Y, Matsukawa N, Yamamoto T, et al. Metabolic profiling of Alzheimer's disease brains. Sci Rep. 2013;3:2364.

28. Seidl R, Beninati S, Cairns N, Singewald N, Risser D, Bavan H, et al. Polyamines in frontal cortex of patients with down syndrome and Alzheimer disease. Neurosci Lett. 1996;206(2-3):193-5.
29. McKhann G, Drachman D, Folstein M, Katzman R, Price D, Stadlan EM. Clinical diagnosis of Alzheimer's disease: Report of the NINCDSADRDA Work Group under the auspices of Department of Health and Human Services Task Force on Alzheimer's Disease. Neurology. 1984;34(7):939-44.

30. Petersen RC, Morris JC. Mild cognitive impairment as a clinical entity and treatment target. Arch Neurol. 2005;62(7):1160-3; discussion 1167.

31. Roth M, Tym E, Mountjoy CQ, Huppert FA, Hendrie H, Verma S, et al. CAMDEX. A standardised instrument for the diagnosis of mental disorder in the elderly with special reference to the early detection of dementia. Br J Psychiatry. 1986;149:698-709.

32. Folstein MF, Folstein SE, McHugh PR. "Mini-mental state". A practical method for grading the cognitive state of patients for the clinician. J Psychiatr Res. 1975;12(3):189-98.

33. Graham SF, Chevallier OP, Elliott CT, Hölscher C, Johnston J, McGuinness B, et al. Untargeted Metabolomic Analysis of Human Plasma Indicates Differentially Affected Polyamine and L-Arginine Metabolism in Mild Cognitive Impairment Subjects Converting to Alzheimer's Disease. PLoS One. 2015;10(3):e0119452.

34. Luo J, Mohammed I, Wärmländer SK, Hiruma Y, Gräslund A, Abrahams JP. Endogenous polyamines reduce the toxicity of soluble A $\beta$ peptide aggregates associated with Alzheimer's disease. Biomacromol. Biomacromolecules. 2014;15(6):1985-91.

35. Yang Y, Chen T, Zhu S, Gu X, Jia X, Lu Y, et al. Two macrocyclic polyamines as modulators of metal-mediated $\mathrm{A} \beta 40$ aggregation. Integr Biol (Camb). 2015;7(6):655-62. 